Aus dem ${ }^{1)}$ Institut für Tierzucht und Haustiergenetik, Göttingen, ${ }^{2)}$ Institut für Tierzucht und Tierverhalten, Mariensee, ${ }^{3)}$ Institut für Fleischerzeugung und Vermarktung der BAFF, Kulmbach

\author{
WORAWUT CHAINETR ${ }^{1)}$, PETER GLODEK ${ }^{1)}$, HORST BRANDT ${ }^{1)}$, \\ BURCHARDT MÖLLERS ${ }^{1,}$, MARTINA HENNING ${ }^{2)}$, ERHARD KALLWEIT ${ }^{2)}$ \\ und KLAUS FISCHER ${ }^{3)}$
}

\title{
Systematische Gebrauchskreuzung als Möglichkeit der Erhaltung vom Aussterben bedrohter Landschweinrassen
}

\author{
Herrn Prof. Drs. h. c. Franz Pirchner PhD, zum 75. Geburtstag gewidmet
}

\begin{abstract}
Summary
Title of the paper: Systematic crossbreeding as a tool to conserve endangered pig breeds

Live conservation of purebred populations of old endangered pig breeds requires high financial subsidies because they are very inferior to actual commercial hybrids in overall economy. But subsidies may be considerably reduced if the endangered breeds can be integrated into regional crossbreeding schemes adapted to ecological production niches or designed for specific quality products. The aim of this study at the experimental station Relliehausen of Göttingen University was to compare the performance of two crossbred sows with the endangered breeds Saddleback (DS) and Bentheimer (BB) as dams sire (and LW/LR as dams dam) with commercial LW/LR-crossbred sows in their litter performance as well as meat production and quality traits of their progeny from the same PI(NN)- and (PI*HA)-terminal sires. 478 litters with 1,384 fattened and 1,037 carcass graded progeny of which about 600 underwent detailed carcass value and meat quality evaluations at a loin cross section and 48 with an additional sensoric test. Results show that in litter performance DS crossbred sows were comparable to LW/LR commercial sows but BB crossbred sows weaned 0.66 pigs less per litter. In FOM grading no significant differences were found between the three sow groups as well as between the two different terminal sires. This also applied for most meat quality traits but in intramuscular fat content unexpectedly the control pigs were the best and significantly superior to the BB-progeny. The typical highly significant quality differences due to the "Hampshire effect" were found between progeny of the (PI*HA)-boar as compared to PI-progeny. In a calculated overall economic value DS-progeny were 4 - 5 DM and BB-progeny about 8 DM per pig inferior to the commercial controls. These differences are only $1 / 5$ to $1 / 10$ of the purebred inferiority of the endangered breeds. These results should be confirmed by a field trial under more extensive conditions (e.g. outdoor sow- and ecological fattener management).
\end{abstract}

$\underline{\text { Key Words: }}$ conservation of endangered pig breeds, commercial crossbreeding, litter performance, meat production, meat quality

\section{Zusammenfassung}

Die Lebenderhaltung alter Landrassen in Reinzucht, die marktüblichen Hybriden wirtschaftlich stark unterlegen sind, erfordert hohe Förderungsbeträge aus öffentlichen Mitteln. Diese könnten wesentlich gesenkt werden, wenn bedrohte Landrassen so in sinnvolle Gebrauchskreuzungsprogramme eingebunden werden, dass vermarktungsfähige Produkte entstehen, die möglichst kostendeckend erzeugt werden können. Dies war die Zielsetzung eines Versuches auf dem Versuchsbetrieb Relliehausen der Universität Göttingen, in welchem Kreuzungssauen der bedrohten Landrassen Deutsche Sattelschweine (DS) und Bunte Bentheimer (BB) mit weißen Intensivmutterrassen (LW, DL) auf ihre Zuchtleistungen und die Fleischleistung und -qualität ihrer Nachkommen aus der Anpaarung mit marktüblichen Vaterrassenebern geprüft wurden. Es gingen 478 Würfe mit 1.384 gemästeten und 1.037 geschlachteten Nachkommen, von denen 600 Kotelettproben analysiert werden konnten, in den Versuch ein. Die Ergebnisse zeigen, dass Kreuzungssauen mit DS etwa gleiche Aufzuchtleistungen aufwiesen wie marktübliche Hybridsauen, die von BB aber 0,66 Ferkel pro Wurf weniger absetzten. In der Klassifizierung gab es für DS- und BB-Endprodukte keine signifikanten Unterschiede zu marktüblichen Hybriden, für die wichtigsten 
Fleischbeschaffenheitskriterien gilt das gleiche. In der Gesamtwirtschaftlichkeit lagen die DS-Sauen um 4 - 5 DM und die BB-Sauen um etwa 8 DM je Mastschwein unter der marktüblicher Hybridschweine. Dieser Versuch sollte jedoch unter extensiven Haltungsverhältnissen, z.B. bei outdoor-Sauenhaltung mit langsamer Mast in Ökobetrieben, wiederholt werden, unter denen die Nachkommen der bedrohten Rassen möglicherweise noch besser abschneiden würden.

Schlüsselwörter: Erhaltung bedrohter Landschweinerassen, Gebrauchskreuzung, Zuchtleistung, Fleischleistung, Fleischbeschaffenheit

1 . Einleitung

Die wirtschaftliche Entwicklung nach dem zweiten Weltkrieg, vor allem die Forderung des Marktes nach möglichst einheitlicher Ware mit hohem Fleischanteil (SPONENBERG, 1994) wie auch einheitliche Agrarpreise und Qualitätsnormen, gekoppelt mit der zunehmenden Effizienz der Tierzucht, diesen gerecht zu werden (SIMON und SCHULTE-COERNE, 1979), haben zu einer Verringerung der Vielfalt alter Landrassen und der Einfuhr neuer Leistungsrassen geführt. Einige Rassen wie Weideschweine und Rotbunte sind völlig verschwunden, andere nur noch in geringen Beständen vorhanden. $\mathrm{Zu}$ den letzteren gehören vor allem die verschiedenen Sattelschweinschläge und das Bunte Bentheimer Schwein.

In den letzten 15 - 20 Jahren hat das Interesse an der Erhaltung alter gefährdeter Nutztierrassen zugenommen, sei es, um einer weiteren Verarmung der Rassenvielfalt und damit dem Verlust eventuell einmal wichtiger Gene entgegenzuwirken, sei es aus dem Wunsch heraus, regional bedeutsame Kulturgüter zu bewahren.

Gegenstand dieser Untersuchung, die im Rahmen eines EU-Projektes zur Spermakryokonservierung bedrohter europäischer Schweinerassen (OLLIVIER et al., 2001) stattfand, sind die ursprünglich in drei verschiedenen Schlägen geführten deutschen Sattelschweine und die Bunten Bentheimer Schweine. Da diese Rassen seit Jahren die am stärksten vom Aussterben bedrohten deutschen Schweinerassen sind, wird ihre Erhaltung in kleinen Lebendpopulationen von mehreren Bundesländern finanziell gefördert, und deshalb wurden sie auch in das europäische Kryokonservierungsprojekt eingebracht (GLODEK, 2000).

Eine dauerhafte Sicherung dieser beiden in Reinzucht leistungsmäßig nicht mehr wettbewerbsfähigen Landrassen (zu fettwüchsig und daher nicht geeignet für intensive Haltungsformen) ist aber nur möglich, wenn für sie im Rahmen regionaler Qualitätsoder Naturschutzprogramme eine sich ökonomisch einigermaßen selbst tragende Aufgabe gefunden werden kann, die wesentlich mehr Landwirte motiviert, diese Rassen weiterhin zu halten. Als Beispiel dafür gilt eine Markenfleischerzeugergemeinschaft in Schwäbisch-Hall, die Kreuzungsprodukte von Sattelschweinsauen mit fleischreichen Pietrainebern, z.T. aus ökologischer Haltung, zu besser bezahlten Spezialfleischwaren verarbeitet und vermarktet (BÜHLER, 1996).

Die Zielsetzung dieser Untersuchung war, Gebrauchskreuzungsprodukte mit Sattelund Bentheimer Schweinen zu prüfen, die in nachhaltiger Produktion, eventuell in regionalen Nischenprogrammen mit natur- und umweltschonendem oder touristischem Image, zu kostendeckenden Preisen vermarktet werden können. Damit würden einerseits die Abhängigkeit von direkten staatlichen Fördermitteln reduziert und zum anderen die Populationsgrößen der zu erhaltenden Landrassen über die akute Bedrohungsgrenze hinaus gesteigert werden können. 


\section{$2 . \quad$ Material und Methoden}

Der Versuch fand mit drei verschiedenen Sauengruppen auf dem Versuchsgut Relliehausen der Universität Göttingen statt, die mit den gleichen Ebern zur Erzeugung von Drei- und Vierrassenendprodukten im Natursprung angepaart wurden. Als Kontrolle (33) dienten die betriebsüblichen Einfach- und z.T. Wechselkreuzungen zwischen Landrasse und Large White auf BHZP- Grundlage (Linien 01 und 03). Verglichen wurden diese Sauen mit den Versuchssauen 71 (DS-Sperma * F1-Sautyp 21) und 72 (BB-Sperma*F1-Sautyp 21), die auf dem Versuchsbetrieb mithilfe des Spermas von je 6 DS- und BB- Ebern aus dem EU-Projekt GENRES 012 erzeugt wurden. Die als Endproduktväter eingesetzten Eber entstammten den beiden auf dem Versuchsgut gezüchteten Vaterkombinationen (PI*HA)-Wechselkreuzung und PI(NN)-Reinzucht. Die Mast erfolgte in gemischtgeschlechtlichen Gruppen von 6 bzw. 12 Tieren im Mastabschnitt von 25 - 110 kg. Die Anzahl der Tiere in den einzelnen Versuchsgruppen ist der folgenden Tabelle 1 zu entnehmen.

Tabelle 1

Anzahl der Würfe, der Mastschweine, der geschlachteten Schweine und der Kotelettproben für den sensorischen Test nach Vater- und Mutterlinien (Number of litters, fattened, carcass graded and cross sectioned progeny in sire and dam groups)

\begin{tabular}{|c|c|c|c|c|c|c|c|}
\hline \multirow[b]{2}{*}{ Deckeber } & \multicolumn{2}{|c|}{33} & \multicolumn{2}{|c|}{71} & \multicolumn{2}{|c|}{72} & \multirow[t]{2}{*}{ Summe } \\
\hline & $\mathrm{PI}(\mathrm{NN})$ & PI*HA & $\mathrm{PI}(\mathrm{NN})$ & $\mathrm{PI}^{*} \mathrm{HA}$ & $\mathrm{PI}(\mathrm{NN})$ & PI*HA & \\
\hline Anzahl Würfe & 136 & 158 & 50 & 41 & 44 & 49 & 478 \\
\hline Anzahl & 407 & 470 & 116 & 118 & 160 & 113 & 1.384 \\
\hline Mastschweine & & & & & & & \\
\hline Anzahl & 277 & 296 & 110 & 110 & 140 & 104 & $\left.1.037^{*}\right)$ \\
\hline $\begin{array}{l}\text { geschlachteter } \\
\text { Schweine }\end{array}$ & & & & & & & \\
\hline Anzahl Kotelett- & $'=4$, & $'=4$, & $'=4$, & $'=4$, & $'=4$, & $'=4$, & $'=24$, \\
\hline $\begin{array}{l}\text { proden } \\
\text { rischen Test }\end{array}$ & $\mapsto=4$ & $\mapsto=4$ & $\mapsto=4$ & $\mapsto=4$ & $\mapsto=4$ & $\mapsto=4$ & $\mapsto=24$ \\
\hline
\end{tabular}

Die Auswertung der Wurfleistung erfolgte mit Hilfe des SAS-Programmpaketes Version 6.12 nach dem Statistischen Modell 1.

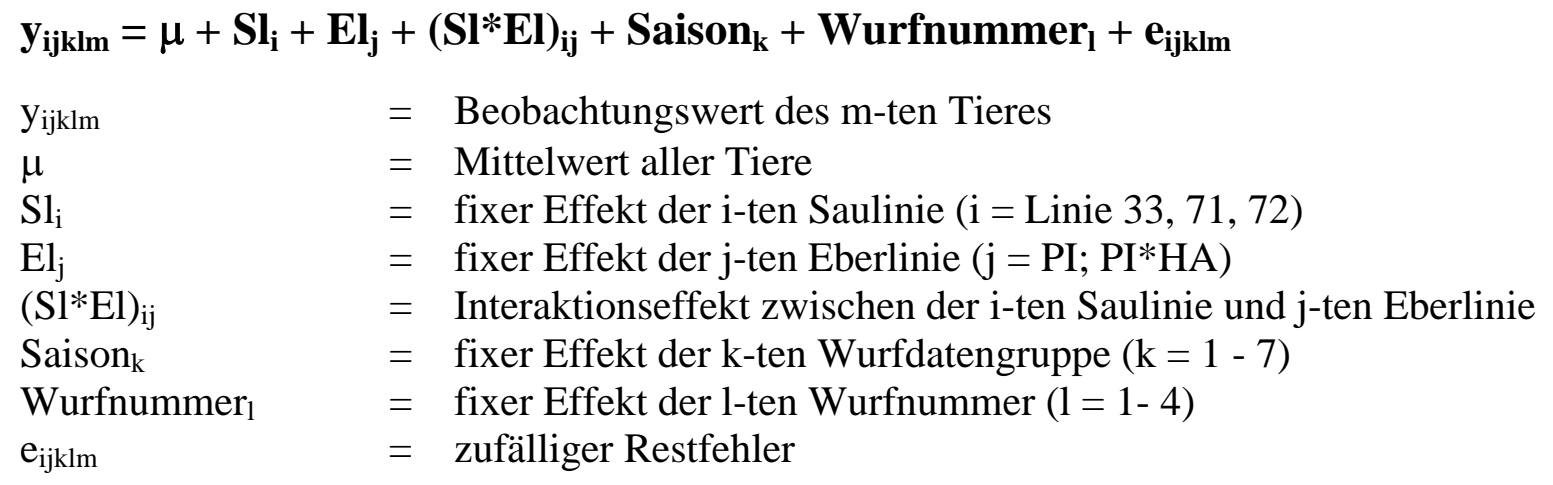

Die Auswertung der individuellen Mastleistungen erfolgte nach Modell 2.

$\mathbf{y}_{\mathrm{ijklm}}=\mu+\mathrm{Sl}_{\mathrm{i}}+\mathrm{El}_{\mathbf{j}}+\left(\mathrm{Sl} \mathbf{E}^{*}\right)_{\mathrm{ij}}+\operatorname{Sex}_{\mathrm{k}}+\operatorname{Saison}_{\mathrm{l}}+\mathrm{KOV}(\mathrm{GA})+\mathbf{e}_{\mathrm{ijklm}}$

Effekte wie im Modell 1, zusätzlich

Sex $_{\mathrm{k}} \quad=$ fixer Effekt des Geschlechts ( $\mathrm{k}=$ Börge und Sauen)

$\operatorname{KOV}(\mathrm{GA}) \quad=$ Kovariable Gewichtsabschnitt der Mast 
Die Schlachtung erfolgte an 43 Schlachttagen im Zeitraum von September 1998 bis November 1999. Die fertigen Mastschweine wurden montags zum Göttinger Schlachthof transportiert (ca 1 Stunde Fahrt) und dort sofort nach Ankunft geschlachtet. Am Schlachttag wurde neben dem FOM-Protokoll der pH-Wert 45 Minuten p.m. zwischen 13. und 14. Rippe erhoben. 24 h p.m. wurden an der gekühlten rechten Hälfte hängend folgende Merkmale bestimmt: Hälftengewicht, Länge, $\mathrm{pH}_{24}$, Leitfähigkeit, Rückenund Seitenspeckdicke, Schinken- und Bauchbeurteilung (1-5 Punkte). Außerdem wurde ein Kotelettfoto zwischen der 13. und 14. Rippe gemacht, an welchem die Fleisch- und Fettfläche sowie das Verhältnis aus beiden und die Speckdicke über dem Kotelett gemessen wurden. Am frischen Hälftenanschnitt wurde der Göfo-Wert gemessen. Eine Kotelettprobe von 450g wurde im Fleischlabor der FAL Mariensee auf Gehaltsmerkmale untersucht.

Nach einer Vorkorrektur für monatlich gemittelte Schlachttage erfolgte eine SASAuswertung nach Modell 2 ohne den Saisoneffekt und mit dem Zweihälftengewicht als Kovariable. Die Schlachtung für den sensorischen Test erfolgte an 10 Schlachttagen im Zeitraum von Mai bis August 2000. Die sensorische Prüfung wurde von 6 Testpersonen der Bundesanstalt für Fleischforschung (BAFF), Kulmbach durchgeführt. Bewertet wurde die Ausprägung der Merkmale „Saftigkeit“, „Zartheit“, „Aroma“ und „Gesamteindruck“. Die Punkteskala reichte von 1 für die schlechteste bis 6 für die beste Beurteilung. Es erfolgte eine SAS-Auswertung nach Modell 2 ohne den Saisoneffekt und die Kovariable, aber mit den zusätzlichen fixen Effekten Testtag und Testperson.

3. $\quad$ Ergebnisse

Die LS-Mittelwerte (Standardfehler) für die Aufzuchtleistung sind in Tabelle 2 nach Mutter- und Vaterlinien dargestellt.

Tabelle 2

LS-Mittelwerte (Standardfehler) für die Zuchtleistung nach Mutter- und Vaterlinien sowie Gesamtmittel und Reststandardabweichung (LS-means (standard errors) for litter performance in dam- and sire groups)

\begin{tabular}{c|ccc|cc|cc}
\hline Merkmale & \multicolumn{3}{c|}{ Mutterlinien } & \multicolumn{2}{c}{ Vaterlinien } & \multicolumn{2}{c}{ Insgesamt } \\
& 33 & 71 & 72 & PI(NN) & PI*HA & Mittelwert & SD \\
\hline Gesamtgeborene & $11,11^{\mathrm{A}}$ & $11,00^{\mathrm{A}}$ & $9,55^{\mathrm{B}}$ & 10,46 & 10,66 & 10,79 & 2,84 \\
Ferkel je Wurf & $(0,17)$ & $(0,31)$ & $(0,30)$ & $(0,22)$ & $(0,22)$ & & \\
Abgesetzte Ferkel je & 8,36 & 8,18 & 7,70 & 7,96 & 8,19 & 8,21 & 2,77 \\
Wurf & $(0,17)$ & $(0,30)$ & $(0,29)$ & $(0,21)$ & $(0,21)$ & & \\
Mittleres & 1,63 & 1,62 & 1,69 & 1,65 & 1,65 & 1,64 & 0,26 \\
Geburtsgewicht der & $(0,02)$ & $(0,03)$ & $(0,03)$ & $(0,02)$ & $(0,02)$ & & \\
Ferkel & & & & & & \\
Mittleres Absatz- & $7,31^{\mathrm{a}}$ & 7,61 & $7,71^{\mathrm{b}}$ & 7,53 & 7,55 & 7,44 & $(0,50$ \\
gewicht der Ferkel & $(0,09)$ & $(0,16)$ & $(0,16)$ & $(0,12)$ & $(0,12)$ & &
\end{tabular}

Tabelle 2 zeigt, dass die BB-Kreuzungssauen signifikant weniger gesamtgeborene Ferkel je Wurf als die beiden anderen Mutterlinien hatten, während bei abgesetzten Ferkeln je Wurf und mittlerem Geburtsgewicht des Wurfes zwischen den Mutterlinien keine Unterschiede auftraten. Die BB-Sauen hatten ein schwachsignifikant besseres mittleres Absatzgewicht des Wurfes als die Kontrollsauen. Die Vaterlinien hatten keine signifikanten Effekte auf die Wurfmerkmale.

Die LS-Mittelwerte (Standardfehler) für die Zunahmen je Lebenstag und im Mastabschnitt sind in Tabelle 3 nach Mutter- und Vaterlinien dargestellt. 
Tabelle 3 zeigt, dass die BB-Nachkommen hoch signifikant schlechtere Zunahmen als die der beiden anderen Mutterlinien hatten. Bei den Vaterlinien vererbten die PI*HAKreuzungseber hoch signifikant höhere Zunahmen als PI(NN)-Eber.

Tabelle 3

LS-Mittelwerte (Standardfehler) für individuelle Zunahmen nach Mutter- und Vaterlinien sowie Gesamtmittel und Reststandardabweichung (LS-means (standard errors) for individual growth in progeny groups of sire and dam lines)

\begin{tabular}{|c|c|c|c|c|c|c|c|}
\hline \multirow[t]{2}{*}{ Merkmale } & \multicolumn{3}{|c|}{ Mutterlinien } & \multicolumn{2}{|c|}{ Vaterlinien } & \multicolumn{2}{|c|}{ Insgesamt } \\
\hline & 33 & 71 & 72 & PI(NN) & PI*HA & Mittelwert & SD \\
\hline $\begin{array}{l}\text { Lebenstags- } \\
\text { zunahme }\end{array}$ & $538^{\mathrm{A}}(1)$ & $535^{\mathrm{A}}(2)$ & $520^{\mathrm{B}}(2)$ & $524^{\mathrm{A}}(2)$ & $538^{\mathrm{B}}(2)$ & 535 & 34 \\
\hline $\begin{array}{l}\text { Tageszunahme im } \\
\text { Abschnitt (g) }\end{array}$ & $668^{\mathrm{A}}(2)$ & $652^{\mathrm{Ba}}(4)$ & $641^{\mathrm{Bb}}(4)$ & $644^{\mathrm{A}}(2)$ & $663^{\mathrm{B}}(3)$ & 661 & 56 \\
\hline
\end{tabular}

In Tabelle 4 sind die LS-Mittelwerte (Standardfehler) für die wichtigsten Schlachtkörper- und Fleischbeschaffenheitsmerkmale nach Mutter- und Vaterlinien wiedergegeben. Dabei wurden am Schlachttag 1.037 Tiere bewertet und 24 h p.m. 599 bis 638 angeschnittene Hälften.

Die Zahlen in Tabelle 4 belegen, dass die BB-Nachkommen in der Nettozunahme signifikant schlechter sind, während in der Ausschlachtung BB- und DS-Nachkommen besser als der Standard sind, jedoch die PI*HA-Kreuzungseber 13g höhere Nettotageszunahmen und $0,79 \%$ schlechtere Ausschlachtung vererben. Im bezahlungsrelevanten FOM-Fleischanteil sind weder Mutter- noch Vaterrassen signifikant verschieden. Aber die DS- und BB-Nachkommen wie die der PI(NN)-Eber zeigten höhere FOM-Speckmaße, was in den Rücken- und Seitenspeckdicken und im Fleisch-Fettverhältnis nicht der Fall ist, wohl aber in der Fettfläche über dem Kotelett. In der Länge waren die Nachkommen der Kontrollsau und des PI*HA-Kreuzungsebers den anderen Gruppen signifikant überlegen. In der Rückenmuskelfläche waren die DS- und BB-, wie die PI(NN)- Nachkommen signifikant besser als die anderen Gruppen. In der Schinkenform wurden die BB-Nachkommen signifikant besser bewertet und im Bauch die der Kontrollsau 33. Unerwartet ist der Befund, dass im geschmacksbestimmenden intramuskulären Fettgehalt die Nachkommen der Kontrollsau leicht besser als die der BB-Sau und diese beiden den DS-Nachkommen hochsignifikant überlegen waren. Letztere hatten auch den höchsten Wassergehalt des Fleisches, womit sie signifikant über den BB-Nachkommen lagen. Diese Zusammenhänge spiegeln sich auch in der spätpostmortalen $\mathrm{LF}_{24}$ wider, während im frühpostmortalen pH45 alle Gruppen auf gleich hohem Niveau lagen. Gering wenn auch schwach signifikant waren die Unterschiede zwischen den Sauengruppen im Reflexions- und Göfowert. Zwischen den Vaterlinien gab es die typischen Unterschiede, die auf den Hampshireanteil im PI*HAEber zurückzuführen sind: Hoch signifikant höhere Wasser- und niedrigere Proteingehalte wie End-PH-Werte bei den Nachkommen des HA-Kreuzungsebers, die allerdings einen hoch signifikant höheren intramuskulären Fettgehalt aufwiesen als die der PI-Eber.

Untersuchungen über sensorische Qualitätsmerkmale des Fleisches zeigt die Tabelle 5. Sie veranschaulicht, dass die Nachkommen der BB-Sau am besten und die der Kontrollsau am schlechtesten in Saftigkeit und Zartheit des Fleisches beurteilt wurden, aber diesen berechneten Differenzen fehlte die Signifikanz $(\mathrm{p}<0,20)$. Die Nachkom- 
men der DS-Sauen bekamen die besten Aroma-Noten, aber auch das konnte mit diesen Probenzahlen nicht statistisch abgesichert werden.

Tabelle 4

LS-Mittelwerte (Standardfehler) für Schlachtkörper- und Fleischbeschaffenheitsmerkmale nach Mutter- und Vaterlinien (LS-means (standard errors) for carcass and meat quality traits in progeny groups of sire and dam lines)

\begin{tabular}{|c|c|c|c|c|c|c|c|}
\hline \multirow[t]{2}{*}{ Merkmale } & \multicolumn{3}{|c|}{ Mutterlinien } & \multicolumn{2}{|c|}{ Vaterlinien } & \multicolumn{2}{|c|}{ Insgesamt } \\
\hline & 33 & 71 & 72 & $\mathrm{PI}(\mathrm{NN})$ & PI*HA & Mittelwert & SD \\
\hline $\begin{array}{l}\text { Nettozunahme } \\
\text { (g / Tag) }\end{array}$ & $\begin{array}{c}432^{\mathrm{A}} \\
(1)\end{array}$ & $\begin{array}{c}430^{\mathrm{A}} \\
(2)\end{array}$ & $\begin{array}{c}418^{\mathrm{B}} \\
(2)\end{array}$ & $\begin{array}{c}420^{\mathrm{A}} \\
(1)\end{array}$ & $\begin{array}{c}433^{\mathrm{B}} \\
(1)\end{array}$ & 430 & 32,96 \\
\hline $\begin{array}{c}\text { Ausschlachtung } \\
\text { (\%) }\end{array}$ & $\begin{array}{l}78,76^{\mathrm{A}} \\
(0,07)\end{array}$ & $\begin{array}{l}79,14^{\mathrm{B}} \\
(0,11)\end{array}$ & $\begin{array}{l}79,29^{\mathrm{B}} \\
(0,11)\end{array}$ & $\begin{array}{l}79,38^{A} \\
(0,08)\end{array}$ & $\begin{array}{l}78,75^{\mathrm{B}} \\
(0,08)\end{array}$ & 78,95 & 1,66 \\
\hline $\begin{array}{l}\text { FOM-Fleischanteil } \\
\text { (\%) }\end{array}$ & $\begin{array}{l}56,56 \\
(0,11)\end{array}$ & $\begin{array}{l}56,38 \\
(0,17)\end{array}$ & $\begin{array}{l}56,22 \\
(0,16)\end{array}$ & $\begin{array}{l}56,25 \\
(0,12)\end{array}$ & $\begin{array}{l}56,52 \\
(0,13)\end{array}$ & 56,36 & 2,41 \\
\hline FOM-Speckmaß (mm) & $\begin{array}{l}15,57^{\mathrm{A}} \\
(0,11)\end{array}$ & $\begin{array}{l}16,25^{\mathrm{B}} \\
(0,17)\end{array}$ & $\begin{array}{l}16,26^{\mathrm{B}} \\
(0,16)\end{array}$ & $\begin{array}{l}16,35^{\mathrm{A}} \\
(0,12)\end{array}$ & $\begin{array}{l}15,70^{\mathrm{B}} \\
(0,13)\end{array}$ & 16,01 & 2,46 \\
\hline FOM-Fleischmaß (mm) & $\begin{array}{l}58,87^{\mathrm{A}} \\
(0,23)\end{array}$ & $\begin{array}{l}61,02^{\mathrm{B}} \\
(0,35)\end{array}$ & $\begin{array}{l}60,17^{\mathrm{B}} \\
(0,33)\end{array}$ & $\begin{array}{l}60,76^{\mathrm{A}} \\
(0,24)\end{array}$ & $\begin{array}{l}59,28^{\mathrm{B}} \\
(0,26)\end{array}$ & 59,83 & 5,15 \\
\hline Schlachtkörperlänge (cm) & $\begin{array}{l}99,10^{A} \\
(0,18)\end{array}$ & $\begin{array}{l}98,08^{\mathrm{B}} \\
(0,17)\end{array}$ & $\begin{array}{l}98,04^{\mathrm{B}} \\
(0,16)\end{array}$ & $\begin{array}{l}98,07^{\mathrm{A}} \\
(0,13)\end{array}$ & $\begin{array}{l}98,74^{\mathrm{B}} \\
(0,15)\end{array}$ & 98,25 & 2,39 \\
\hline Rückenspeckdicke (mm) & $\begin{array}{l}23,55^{\mathrm{A}} \\
(0,21)\end{array}$ & $\begin{array}{l}24,92^{\mathrm{B}} \\
(0,21)\end{array}$ & $\begin{array}{l}23,60^{\mathrm{A}} \\
(0,20)\end{array}$ & $\begin{array}{l}24,11 \\
(0,16)\end{array}$ & $\begin{array}{l}23,94 \\
(0,18)\end{array}$ & 24,35 & 2,89 \\
\hline $\begin{array}{l}\text { Seitenspeckdicke } \\
\text { (mm) }\end{array}$ & $\begin{array}{l}19,46 \\
(0,34)\end{array}$ & $\begin{array}{l}20,39 \\
(0,33)\end{array}$ & $\begin{array}{l}19,86 \\
(0,32)\end{array}$ & $\begin{array}{l}20,15 \\
(0,26)\end{array}$ & $\begin{array}{l}19,66 \\
(0,29)\end{array}$ & 20,42 & 4,63 \\
\hline Speckdicke über dem & $11,41^{\mathrm{A}}$ & $12,19^{\mathrm{B}}$ & $12,14^{\mathrm{B}}$ & $12,19^{\mathrm{a}}$ & $11,63^{\mathrm{b}}$ & 12,37 & 2,87 \\
\hline Kotelett (mm) & $(0,21)$ & $(0,21)$ & $(0,20)$ & $(0,16)$ & $(0,18)$ & & \\
\hline $\begin{array}{l}\text { Muskelfläche } \\
\left(\mathrm{cm}^{2}\right)\end{array}$ & $\begin{array}{l}47,84^{A} \\
(0,31)\end{array}$ & $\begin{array}{l}50,64^{\mathrm{Ba}} \\
(0,30)\end{array}$ & $\begin{array}{c}49,83^{\mathrm{Bb}} \\
(0,21)\end{array}$ & $\begin{array}{l}50,50^{\mathrm{A}} \\
(0,23)\end{array}$ & $\begin{array}{l}48,36^{\mathrm{B}} \\
(0,26)\end{array}$ & 49,41 & 4,12 \\
\hline $\begin{array}{c}\text { Fettfläche } \\
\left(\mathrm{cm}^{2}\right)\end{array}$ & $\begin{array}{l}16,47^{\mathrm{A}} \\
(0,22)\end{array}$ & $\begin{array}{l}18,19^{\mathrm{Ba}} \\
(0,21)\end{array}$ & $\begin{array}{l}17,61^{\mathrm{Bb}} \\
(0,20)\end{array}$ & $\begin{array}{l}17,93^{\mathrm{A}} \\
(0,17)\end{array}$ & $\begin{array}{l}16,91^{\mathrm{B}} \\
(0,18)\end{array}$ & 17,95 & 2,95 \\
\hline $\begin{array}{l}\text { Fleisch:Fett-Verhältnis } \\
\qquad(1: \ldots)\end{array}$ & $\begin{array}{c}0,35 \\
(0,005)\end{array}$ & $\begin{array}{c}0,36 \\
(0,005)\end{array}$ & $\begin{array}{c}0,36 \\
(0,004)\end{array}$ & $\begin{array}{c}0,36 \\
(0,004)\end{array}$ & $\begin{array}{c}0,35 \\
(0,004)\end{array}$ & 0,37 & 0,07 \\
\hline Schinkenpunkte & $\begin{array}{l}2,16^{\mathrm{A}} \\
(0,04)\end{array}$ & $\begin{array}{l}2,11^{\mathrm{A}} \\
(0,04)\end{array}$ & $\begin{array}{l}2,33^{\mathrm{B}} \\
(0,04)\end{array}$ & $\begin{array}{l}2,42^{\mathrm{A}} \\
(0,03)\end{array}$ & $\begin{array}{l}1,99^{\mathrm{B}} \\
(0,04)\end{array}$ & 2,21 & 0,59 \\
\hline Bauchpunkte & $\begin{array}{l}3,07^{A} \\
(0,06)\end{array}$ & $\begin{array}{l}2,83^{\mathrm{B}} \\
(0,06)\end{array}$ & $\begin{array}{l}2,87^{\mathrm{B}} \\
(0,05)\end{array}$ & $\begin{array}{c}2,89 \\
(0,04)\end{array}$ & $\begin{array}{l}2,96 \\
(0,05)\end{array}$ & 2,88 & 0,77 \\
\hline $\begin{array}{c}\text { Wassergehalt des } \\
\text { Fleisches (\%) }\end{array}$ & $\begin{array}{l}75,07^{\mathrm{AB}} \\
(0,05)\end{array}$ & $\begin{array}{l}75,15^{\mathrm{A}} \\
(0,05)\end{array}$ & $\begin{array}{l}74,94^{\mathrm{B}} \\
(0,05)\end{array}$ & $\begin{array}{l}74,86^{\mathrm{A}} \\
(0,04)\end{array}$ & $\begin{array}{l}75,25^{\mathrm{B}} \\
(0,04)\end{array}$ & 75,05 & 0,70 \\
\hline $\begin{array}{l}\text { Intramuskulärer } \\
\text { Fettgehalt (\%) }\end{array}$ & $\begin{array}{l}1,91^{\mathrm{A}} \\
(0,05)\end{array}$ & $\begin{array}{l}1,69^{\mathrm{B}} \\
(0,05)\end{array}$ & $\begin{array}{l}1,87^{\mathrm{A}} \\
(0,04)\end{array}$ & $\begin{array}{l}1,69^{\mathrm{A}} \\
(0,04)\end{array}$ & $\begin{array}{l}1,96^{\mathrm{B}} \\
(0,04)\end{array}$ & 1,82 & 0,62 \\
\hline $\begin{array}{l}\text { Proteingehalt des } \\
\text { Fleisches (\%) }\end{array}$ & $\begin{array}{l}22,13^{\mathrm{A}} \\
(0,07)\end{array}$ & $\begin{array}{l}22,28^{\mathrm{AB}} \\
(0,06)\end{array}$ & $\begin{array}{l}22,44^{\mathrm{B}} \\
(0,06)\end{array}$ & $\begin{array}{l}22,82^{\mathrm{A}} \\
(0,05)\end{array}$ & $\begin{array}{l}21,76^{\mathrm{B}} \\
(0,06)\end{array}$ & 22,31 & 0,89 \\
\hline Reflexionswert & $\begin{array}{l}27,18^{\mathrm{a}} \\
(0,16)\end{array}$ & $\begin{array}{l}26,52^{\mathrm{b}} \\
(0,24)\end{array}$ & $\begin{array}{c}26,72^{\mathrm{ab}} \\
(0,23)\end{array}$ & $\begin{array}{c}26,34^{\mathrm{A}} \\
(0,17)\end{array}$ & $\begin{array}{l}27,28^{\mathrm{B}} \\
(0,18)\end{array}$ & 26,71 & 3,42 \\
\hline$\left[\mathrm{pH}_{45}\right]$ & $\begin{array}{c}6,25 \\
(0,01)\end{array}$ & $\begin{array}{c}6,26 \\
(0,02)\end{array}$ & $\begin{array}{c}6,23 \\
(0,01)\end{array}$ & $\begin{array}{c}6,23 \\
(0,01)\end{array}$ & $\begin{array}{c}6,26 \\
(0,01)\end{array}$ & 6,25 & 0,22 \\
\hline$\left[\mathrm{pH}_{24}\right]$ & $\begin{array}{c}5,42 \\
(0,01)\end{array}$ & $\begin{array}{c}5,42 \\
(0,01)\end{array}$ & $\begin{array}{c}5,42 \\
(0,01)\end{array}$ & $\begin{array}{c}5,44^{\mathrm{A}} \\
(0,004)\end{array}$ & $\begin{array}{c}5,40^{\mathrm{B}} \\
(0,005)\end{array}$ & 5,42 & 0,08 \\
\hline$\left[\mathrm{LF}_{24}\right]$ & $\begin{array}{l}3,49^{A} \\
(0,07)\end{array}$ & $\begin{array}{l}3,78^{\mathrm{B}} \\
(0,07)\end{array}$ & $\begin{array}{l}3,42^{\mathrm{A}} \\
(0,07)\end{array}$ & $\begin{array}{c}3,55 \\
(0,05)\end{array}$ & $\begin{array}{c}3,58 \\
(0,05)\end{array}$ & 3,50 & 0,95 \\
\hline [GÖFO] & $\begin{array}{l}59,69^{A} \\
(0,23)\end{array}$ & $\begin{array}{c}59,11^{\mathrm{AB}} \\
(0,23)\end{array}$ & $\begin{array}{l}58,83^{\mathrm{B}} \\
(0,22)\end{array}$ & $\begin{array}{l}59,27 \\
(0,18)\end{array}$ & $\begin{array}{l}59,15 \\
(0,20)\end{array}$ & 59,18 & 2,98 \\
\hline
\end{tabular}


Tabelle 5

LS-Mittelwerte (Standardfehler) für sensorische Beurteilungsnoten nach Mutter- und Vaterlinien (LS-means (standard errors) for sensoric scores in progeny groups of sire and dam lines)

\begin{tabular}{|c|c|c|c|c|c|c|c|}
\hline \multirow[t]{2}{*}{ Merkmale } & \multicolumn{3}{|c|}{ Mutterlinien } & \multicolumn{2}{|c|}{ Vaterlinien } & \multicolumn{2}{|c|}{ Insgesamt } \\
\hline & 33 & 71 & 72 & PI(NN) & PI*HA & Mittelwert & $\mathrm{SD}$ \\
\hline Saftigkeit & $\begin{array}{c}3,43 \\
(0,10)\end{array}$ & $\begin{array}{c}3,48 \\
(0,10)\end{array}$ & $\begin{array}{c}3,60 \\
(0,09)\end{array}$ & $\begin{array}{c}3,49 \\
(0,07)\end{array}$ & $\begin{array}{c}3,51 \\
(0,07)\end{array}$ & 3,53 & 0,83 \\
\hline Zartheit & $\begin{array}{c}3,52 \\
(0,11)\end{array}$ & $\begin{array}{c}3,70 \\
(0,12)\end{array}$ & $\begin{array}{c}3,76 \\
(0,11)\end{array}$ & $\begin{array}{c}3,75 \\
(0,08)\end{array}$ & $\begin{array}{c}3,58 \\
(0,08)\end{array}$ & 3,65 & 0,97 \\
\hline Aroma & $\begin{array}{c}3,55 \\
(0,10)\end{array}$ & $\begin{array}{c}3,79 \\
(0,11)\end{array}$ & $\begin{array}{c}3,60 \\
(0,09)\end{array}$ & $\begin{array}{c}3,57 \\
(0,07)\end{array}$ & $\begin{array}{c}3,71 \\
(0,07)\end{array}$ & 3,58 & 0,85 \\
\hline Gesamteindruck & $\begin{array}{c}3,45 \\
(0,10)\end{array}$ & $\begin{array}{c}3,60 \\
(0,11)\end{array}$ & $\begin{array}{c}3,66 \\
(0,09)\end{array}$ & $\begin{array}{c}3,62 \\
(0,07)\end{array}$ & $\begin{array}{c}3,52 \\
(0,07)\end{array}$ & 3,52 & 0,83 \\
\hline
\end{tabular}

4.

\section{Diskussion}

In Tabelle 6 wird versucht, die Leistungsunterschiede zwischen den drei Sauengruppen ökonomisch zu bewerten. Dabei werden die Unterschiede in der Wurfleistung mit DM 7,50 je mehr abgesetztes Ferkel und die Mastleistungen mit DM 0,17 je g mehr täglicher Zunahme bewertet. Die Erlösdifferenzen ergeben sich aus den Einstufungen der Schlachtkörper nach der gültigen Nord-West-Bezahlungsmaske in der FOM-Klassifizierung (ARDEN und BROSTHAUS, 1999) bei einem Basispreis von DM 2,50 je kg Schlachtgewicht (mit 56\% FOM-Fleischanteil).

Aus der Summe dieser drei Kriterien ergibt sich, dass die Nachkommen der DS-Kreuzungssau um DM 4,26 und die der BB-Kreuzungsau um DM 7,93 je Mastschwein dem heute am Markt üblichen Standardprodukt unterlegen sind. Da die Qualitätskriterien der verschiedenen Endprodukte sich nur im intramuskulären Fettgehalt, und dort zugunsten der Kontrollschweine, unterschieden, würde eine zusätzliche Bewertung dieses Merkmals (im unteren Tabellenteil), wie von KRIETER (2001) für Markenprogramme vorgeschlagen, die Unterlegenheiten der DS-Nachkommen auf DM 5,44 und der BB-Nachkommen auf DM 8,14 je Mastschwein erhöhen. Unter den auf der Versuchswirtschaft Relliehausen gegebenen Produktionsbedingungen waren die Nachkommen der bedrohten Landrassen BB und DS also insgesamt um etwa 8 bzw. 4 DM pro Mastschwein dem heutigen Standardangebot an deutschen Märkten unterlegen. Dies wäre etwa der Förderungsbetrag der auch dann zu ihrer Erhaltung benötigt würde, wenn es gelänge, die Rassen in ein sinnvolles Mehrfachkreuzungsprojekt mit nachhaltiger Produktion einzubauen. Vergleicht man dies mit den Verlusten, die sich für die Mast von Reinzuchttieren ergeben (aus Prüfdaten von SCHÖN und BRADE, 1996 lassen sich Mindererträge von 30 - 45 DM pro Mastschwein errechnen), so ist die Bedeutung zu erkennen, die geeignete Mehrfachkreuzungen als Nischenprogramme für die Erhaltung bedrohter Landrassen haben können.

Für die Übertragung dieser Ergebnisse auf praktische Verhältnisse sind jedoch aus zwei Gründen weitere Untersuchungen wünschenswert. Erstens ist die Verwendung der beiden bedrohten Landrassen in der Praxis nur als Mutters-Mutter zielführend, denn nur so wäre die Erhaltung von genügend großen Reinzuchtpopulationen für die bedrohten Rassen gesichert. In diesem Versuch wurden diese Rassen aber nur als Mutters-Vater über Besamung geprüft, so dass untersucht werden müsste, ob die reziproken Sauen zu 71 und 72 ähnliche oder möglicherweise sogar bessere Zuchtleistungen bringen. Dies kann nur in Zusammenarbeit mit Reinzuchtbetrieben für DS und BB 
geschehen, da sie die Kreuzungssauen, wahrscheinlich mit Sperma von DL- oder LW(DE)-Ebern, erzeugen müssen, was für die größeren von ihnen eine interessante Einnahme sein könnte, um ihre Existenz zu sichern.

Tabelle 6

Ökonomische Gesamtbewertung der Endprodukte nach Mutterlinien (Overall economic values for progeny groups of dam lines)

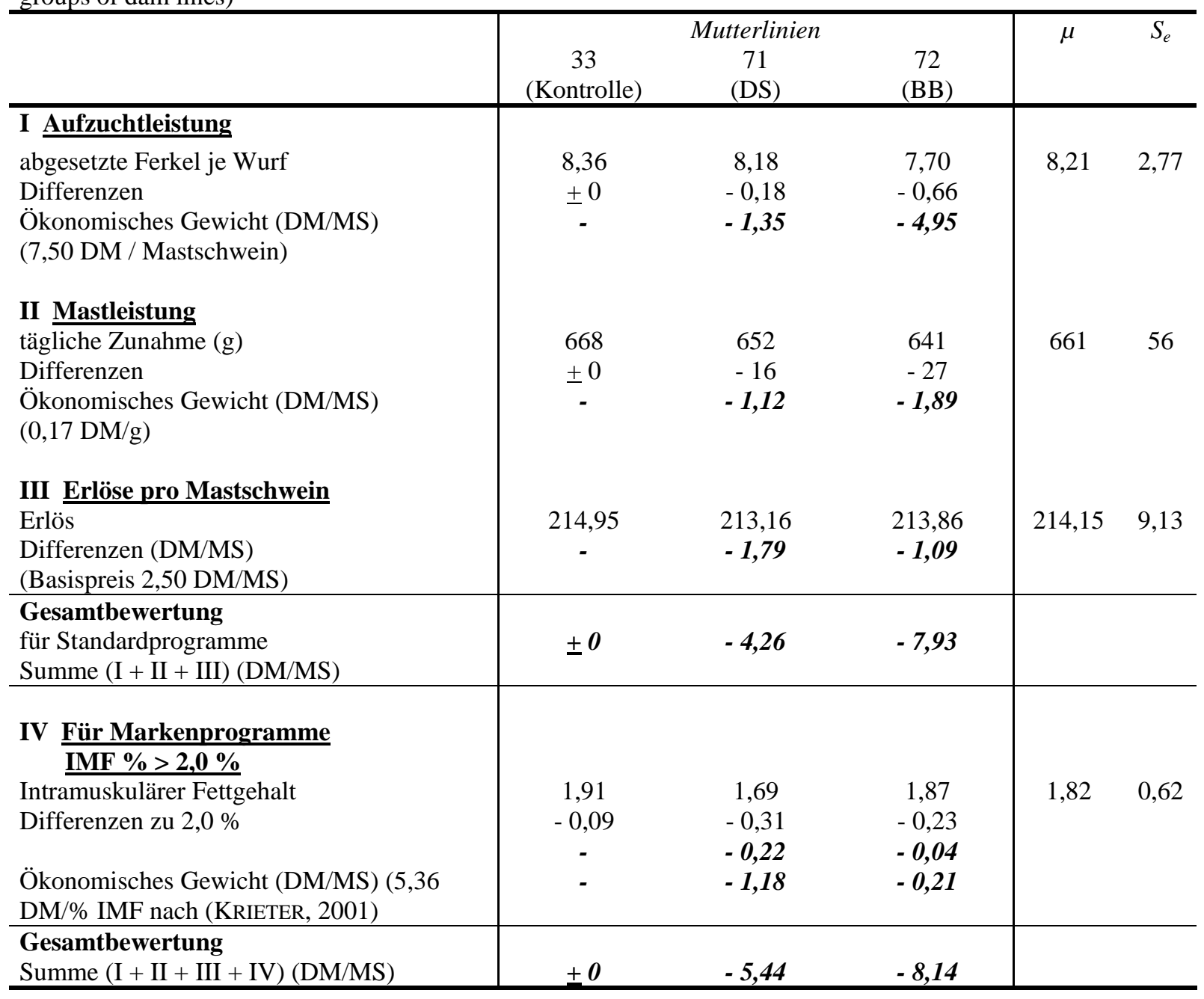

Zweitens wäre zu prüfen, ob diese Kreuzungssauen, besonders die mit BB-Anteil, die unter intensiven Haltungsbedingungen unseres Versuchsbetriebes 0,66 Ferkel pro Wurf weniger absetzten, nicht unter extensiveren Verhältnissen besser abschneiden würden. Eine Wiederholung dieses Versuches mit den „richtigen“ Kreuzungssauen unter praktischen outdoor-Verhältnissen wäre daher erforderlich, um ein endgültiges Urteil zu fällen. Auch wäre ein Vergleich der Mastendprodukte unter noch extensiveren Mastbedingungen (z.B. in Öko-Mastbetrieben) wünschenswert, da dort möglicherweise andere Mast- und Ausschlachtungsergebnisse erreicht würden als auf unserem Versuchsgut.

Die Integration bedrohter Rassen in nachhaltige Produktionsprogramme, auch und gerade in kleinen ökologischen Nischen, erfordert jedoch die Gründung lebensfähiger Erzeugergemeinschaften, wozu - wie das Beispiel aus der nordwestdeutschen Intensivproduktion zeigt - disziplinierte und integrationswillige Betriebe in ausreichender Zahl und Qualität gehören, damit eine beachtenswerte Marktmacht erreicht und kontinuierlich aufrechterhalten wird. Ein Beispiel dafür hat die „Bäuerliche Erzeugergemeinschaft Schwäbisch-Hall“ (BÜHLER, 1996) gegeben, die entscheidend für die zu- 
künftige Lebenderhaltung des in Herdbuch-Reinzucht bereits 1973 ausgestorbenen Schwäbisch-Hällischen Sattelschweins geworden ist. Zumindest für die in vier weiteren Bundesländern versprengten eng verwandten und für eigenständige Programme viel zu kleinen Sattelschweinreste wäre daher anzuraten, zusammen mit dem Schwäbisch-Hällischen Programm die Zuchtplanung in einer bundesweit geführten Herdbuchpopulation zu optimieren. Dies würde allerdings auch eine zwischen den beteiligten Ländern abgestimmte Förderung erfordern.

Noch schwieriger wird es mit einer so regional begrenzten kleinen Population wie dem Bunten Bentheimer Schwein. Es wäre aber durchaus denkbar, dass seine Herdbuchführung und Zuchtbetreuung in einem bundesweit tätigen Verband für bedrohte Schweinerassen (also z.B. dem der Sattelschweine) besser aufgehoben wäre als bei dem regional zuständigen Zuchtverband für marktübliche Schweine, dem sie heute lt. Tierzuchtgesetz von der Obersten Landesbehörde zugeordnet werden. Auch dafür müssten jedoch die zuständigen Landesbehörden die notwendigen administrativen (und möglicherweise finanziellen) Voraussetzungen schaffen.

\section{Literatur}

ARDEN; BROSTHAUS:

Maskenänderung - Wieder ein Alleingang der Schlachtbranche. Top Agrar 8 (1999), S2-S3 BÜHLER, R.:

Das Schwäbisch-Hällische Landschwein - älteste und traditionsreichste Schweinerasse Deutschlands. Selbstverlag (1996)

GLODEK, P.:

Theorie und Praxis der Erhaltung tiergenetischer Ressourcen am Beispiel zweier alter deutscher Schweinerassen. Proc. Symp. zu Ehren von Prof. Dr. D. L. Simon, 11.08.2000, TiHo Hannover

KRIETER, J.:

Ökonomische Zuchtzielkomponenten (Leistungs- und funktionale Merkmale). Proc. 5. Schweineworkshop, Uelzen (2001), 7-17

OLLIVIER L; LABROUE, F.; GLODEK, P.: GANDINI, G.; DELGADO, J.V. (Ed.):

Characterisation and Conservation of Pig Genetic Resources in Europe. EAAP Publ. No. 104 (150 pp) Wageningen Pers., Wageningen 2001

SCHÖN, A.; BRADE, W.:

Alte Schweinerassen im Test. In : Leistungsprüfungen in der Tierproduktion. LWK Hannover, (1996) 46-54 (auch DLZ 3 (1997), 144-148)

SIMON, D.; SCHULTE-COERNE, H.:

Verlust genetischer Alternativen in der Tierzucht - notwendige Konsequenzen. Züchtungskunde, Stuttgart 51 (1979), 332-342

SPONENBERG, D.P.:

What is meant by genetic resources? ALBC News. 11/3 (1994), 2-8

Eingegangen: 15.06.2001

Akzeptiert: 11.12.2001

Anschriften der Verfasser

Prof. Dr. PETER GLODEK,

Dr. WORAWUT CHAINETR,

Dipl.-Agr .ing. BURCHARDT MÖLLERS

Inst. f. Tierzucht und Haustiergenetik

Albert-Thaer-Weg 3

D-37075 Göttingen

e-mail:pglodek@gwdg.de

Dr. KLAUS FISCHER

Inst. f. Fleischforschung und Vermarktung

E.C. Baumann-Str. 20

D-95236 Kulmbach

PD Dr. HORST BRANDT, Univ. Gießen, Institut für Tierzucht und Haustiergenetik

Ludwigstr. 21B

D-35390 Gießen

Prof. Dr. ERHARD KALLWEIT,

Dr. MARTINA HENNING

Inst. für Tierzucht und Tierverhalten

der FAL Mariensee

D-31535 Neustadt

e-mail: mhenning@tvz.fal.de

e-mail: kallweit@tzv.fal.de 